\title{
Approximate analytic solutions to a nonlinear digester problem
}

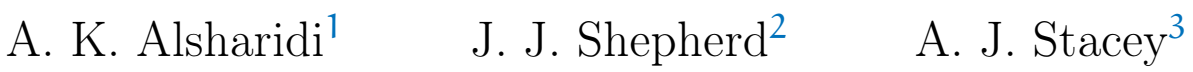 \\ A. A. $\operatorname{Khan}^{4}$
}

(Received 3 March 2020; revised 15 July 2020)

\begin{abstract}
Biological reactors are employed in industrial applications to break down organic waste from a range of sources into components that may be used in other applications. Such reactors may involve complex processes and many components linked by complicated interrelations. These reactions are represented mathematically as nonlinear initial value problems that must be solved numerically. Even smaller systems, more amenable to analytical analysis, require numerical solution methods due to their nonlinearity. We study a simple reactor with only two interacting components - a bacteria consuming a substrate (waste), represented by a $2 \times 2$ autonomous nonlinear initial value problem not solvable analytically. We describe a process to convert this problem to an approximating linear one that can be solved exactly
\end{abstract}

DOI:10.21914/anziamj.v61i0.15196, (C) Austral. Mathematical Soc. 2020. Published 2020-08-30, as part of the Proceedings of the 14th Biennial Engineering Mathematics and Applications Conference. ISSN 1445-8810. (Print two pages per sheet of paper.) Copies of this article must not be made otherwise available on the internet; instead link directly to the DOI for this article. 
to provide a closed form approximate representation of the evolving system. We assess the results of this approach and show they often agree favourably with numerical computations of the original nonlinear problem, although not always.

\section{Contents}

1 Introduction

C230

2 Critical points and physically realistic solutions

C232

3 The linearized system

C234

4 Exact solutions of the linearized system

C235

5 Discussion

C236

\section{Introduction}

Anaerobic digestion involves interactions between bacteria and organic waste (the substrate), in the absence of oxygen. In this process, the bacteria consume substrate, producing biogas, which is useful as fuel. The reaction kinetics of anaerobic digestion have been described by several models of varying complexity. The Anaerobic Digestion Model 1 (ADM1) [2] involves over 30 state variables. A simpler model [3] involves four. Other models vary in complexity $[5,6]$, but for all, analytical mathematical study is very difficult. So, for detailed mathematical analysis, simple models incorporating essential features of the process are required. Here, we study a simple model involving two state variables which involves a Monod (Michaelis-Menten) interaction [4] and is given by the initial value problem

$$
\frac{\mathrm{d} S}{\mathrm{dT}}=\frac{\mathrm{S}_{\mathrm{F}}-\mathrm{S}(\mathrm{T})}{\Theta}-\frac{\mathrm{F}(\mathrm{S}) \mathrm{MX}(\mathrm{T})}{\mathrm{Y}}, \quad \mathrm{S}(0)=\mathrm{S}_{0},
$$




$$
\frac{d X}{d T}=\frac{X_{F}-X(T)}{\Theta}-K_{d} X(T)+F(S) M X(T), \quad X(0)=X_{0},
$$

where

$$
F(S)=\frac{S}{S+K} .
$$

Here, $S(T)$ and $X(T)$ are substrate and bacteria concentrations, respectively at time $T$. The Monod term governing growth rate of bacteria is $F(S)$. The maximum specific growth rate of bacteria is $M, \Theta$ is the hydraulic residence time (the mean time a substrate or bacterium particle is in the system), $K_{d}$ is the bacteria death rate, $\mathrm{Y}$ is the ratio of bacteria to substrate concentration, and $\mathrm{K}$ is the substrate concentration at half maximum specific growth rate. The concentrations of substrate and bacteria entering the system are $S_{F}$ and $X_{F}$, respectively, and $S_{0}$ and $X_{0}$ are initial substrate and bacteria concentrations.

Here, we assume all parameters to be positive. In particular, $X_{F}>0$, that is, the nutrient feed stream contains bacteria. This contrasts with many applications where $X_{F}=0$ [1, p. 382], [7, p. 9]. We work with a dimensionless version of the problem, by introducing dimensionless variables $s, x$ and $t$, and dimensionless parameters $s_{f}$ and $x_{f}$ defined by

$$
\mathrm{S}=\mathrm{Ks}, \quad \mathrm{X}=\mathrm{KYx}, \quad \mathrm{t}=\mathrm{MT}, \quad \mathrm{S}_{\mathrm{F}}=\mathrm{Ks}_{\mathrm{f}}, \quad \mathrm{X}_{\mathrm{F}}=\mathrm{KY \chi _{ \textrm {f } }} .
$$

In terms of the dimensionless quantities, the system (1)-(3) converts to the dimensionless form

$$
\begin{aligned}
& \frac{d s(t)}{d t}=\varpi\left(s_{f}-s(t)\right)-f(s) x(t), \quad s(0)=s_{0}, \\
& \frac{d x(t)}{d t}=\varpi\left(x_{f}-x(t)\right)-\rho x(t)+f(s) x(t), \quad x(0)=x_{0},
\end{aligned}
$$

where

$$
f(s)=\frac{s}{1+s}, \quad \varpi=\frac{1}{\Theta M}, \quad \rho=\frac{K_{d}}{M}, \quad s_{0}=\frac{S_{0}}{K}, \quad x_{0}=\frac{X_{0}}{Y K} .
$$


The difficulty with the dimensionless model, and others, is that their nonlinearity means that any mathematical analysis is restricted to numerical solutions. Numerical solutions have the weakness of being restricted to specific data, and general trends are hard to identify.

In this article, we analyse the model (5)-(6) by approximating it to a linear system which can be readily solved in general terms. This resulting solution has the benefit of giving general expressions for $s(t)$ and $x(t)$ that display the effects of significant parameters. We also investigate the degree to which these expressions approximate the numerical solutions of (5)-(6).

\section{Critical points and physically realistic solutions}

The initial value problem (5)-(7) is nonlinear and is not solvable exactly by analytical methods. Consequently, numerical techniques must be resorted to. However, (5)-(7) is autonomous so standard phase plane techniques may be used to obtain qualitative information about solutions of the system.

In particular, it is a straightforward exercise to show that, for $\varpi$ and $\rho$ positive and $k_{d}, s_{f}$ and $x_{f}$ non-negative, trajectories originating within the closed first quadrant $s \geqslant 0, x \geqslant 0$ of the $(s, x)$ (i.e., phase) plane are totally contained within that quadrant. Thus, solutions of the initial value problem (5)-(7) corresponding to initial values $s_{0} \geqslant 0, x_{0} \geqslant 0$ are non-negative functions for all $t \geqslant 0$.

Here, we are interested in solutions of the problem (5)-(6) that approach a finite limiting state $\left(s^{*}, x^{*}\right)$ in $s>0$ and $x>0$ as $t \rightarrow \infty$. We term such solutions physically realistic solutions.

For each finite critical point $\left(s^{*}, x^{*}\right)$ of the nonlinear system (5)-(6) we associate a solution of this system passing through the point $\left(s_{0}, x_{0}\right)$. When $\left(s_{0}, x_{0}\right)$ lies in the first quadrant, this solution is a physically real solution with $\left(s^{*}, x^{*}\right)$ as a limiting critical point. Note that there may be a number of such critical 
points, and each will correspond to a physically real solution for some initial value $\left(s_{0}, x_{0}\right)$. That is, a critical point may have a domain of attraction.

Thus, the question arises as to how many stable critical points the system (5)(6) has in the first quadrant.

All critical points $\left(\mathrm{s}^{*}, \mathrm{x}^{*}\right)$ are characterised as zeros of the right hand sides of (5) and (6), that is, they are solutions of the system of two equations

$$
\begin{array}{r}
\varpi\left(s_{f}-s\right)-x f(s)=0, \\
\varpi\left(x_{f}-x\right)-\rho x+\chi f(s)=0 .
\end{array}
$$

We first find $x$ in terms of $s$ by adding (8) and (9) and rearranging to give

$$
x=\frac{\varpi\left(s_{f}+x_{f}-s\right)}{b}
$$

where $b=\varpi+\rho$.

We note that if $s_{f}+x_{f}<s^{*}$, then the critical point $\left(s^{*}, x^{*}\right)$ would not lie in the first quadrant. Thus, for $\left(s^{*}, x^{*}\right)$ to be the limit point of a physically real solution it is necessary that

$$
s_{f}+x_{f}>s^{*} .
$$

Substituting for $x$ from equation (10) into equation (8) and using the first of (7) results in a quadratic equation for $s$ :

$$
\mathrm{q}_{2} \mathrm{~s}^{2}+\mathrm{q}_{1} \mathrm{~s}+\mathrm{q}_{0}=0
$$

where

$$
\mathrm{q}_{2}=\mathrm{b}-1, \quad \mathrm{q}_{1}=\mathrm{b}+\mathrm{x}_{\mathrm{f}}-\mathrm{q}_{2} \mathrm{~s}_{\mathrm{f}}, \quad \mathrm{q}_{0}=-\mathrm{b} \mathrm{s}_{\mathrm{f}} .
$$

Finding all real solutions of (12) and the corresponding bacteria concentrations using (10) gives all the critical points $\left(s^{*}, x^{*}\right)$; and those critical points lying in the first quadrant provide limiting states of physically real solutions of (5)-(6). 
The discriminant of (12) is easily shown to be positive for any $b \neq 1$ and zero for $b=1$. So, real distinct solutions of (12) will occur if $b \neq 1$.

Note that since $q_{0} / q_{2}=-b s_{f} /(b-1)$, then if $b>1$ there is one positive and one negative root, while if $\mathrm{b}<1$ there are two positive roots. We discard the negative root, since it will not contribute to a physically realistic critical point.

Of the positive roots, when $b<1$, we find that the larger fails to meet the criterion (11), so it is rejected, while the smaller satisfies (11). Thus, corresponding to each of $b \neq 1$, we have a single critical point of (5)-(6) lying in the first quadrant, $s>0, x>0$. This is given by

$$
\left(s^{*}, x^{*}\right)=\left(\frac{-q_{1}+\sqrt{q_{1}^{2}-4 q_{2} q_{0}}}{2 q_{2}}, \frac{\varpi\left(s_{f}+x_{f}-s_{1}^{*}\right)}{b}\right), \quad b \neq 1 .
$$

When $b=1$, equation (12) becomes $q_{1} s^{*}+q_{0}=0$ resulting in

$$
\left(s^{*}, x^{*}\right)=\left(\frac{s_{f}}{1+x_{f}}, x_{f}+s_{f}\left(1-\frac{1}{1+x_{f}}\right)\right) .
$$

Standard stability analysis based on the Jacobian $J\left(s^{*}, x^{*}\right)$ of the right hand side of (5)-(6) at $\left(s^{*}, x^{*}\right)$ shows that the critical points described above are all stable. In particular, it is straightforward to show that $\operatorname{tr} J\left(\mathrm{~s}^{*}, x^{*}\right)<0$, while $\operatorname{det} J\left(s^{*}, x^{*}\right)>0$, so that if $\left[\operatorname{tr} J\left(s^{*}, x^{*}\right)\right]^{2}-4 \operatorname{det} J\left(s^{*}, x^{*}\right)>0$, then $\left(s^{*}, x^{*}\right)$ is a stable node, while if $\left[\operatorname{tr} J\left(s^{*}, x^{*}\right)\right]^{2}-4 \operatorname{det} J\left(s^{*}, x^{*}\right)<0$, then $\left(s^{*}, x^{*}\right)$ is a stable spiral point.

\section{The linearized system}

Here, we use the critical point of the nonlinear system to construct a linearized system with the same critical point as the nonlinear system. This linearized system is constructed by replacing $f(s)$ in $(5)-(6)$ by $f\left(s^{*}\right)$, its value at a 
known stable, physically realistic, limiting state $s=s^{*}$, when such a limiting state exists. Using this replacement, we obtain the linear system

$$
\begin{aligned}
& \frac{d s}{d t}=\varpi\left(s_{f}-s\right)-f\left(s^{*}\right) x, \quad s(0)=s_{0}, \\
& \frac{d x}{d t}+\alpha x=\varpi x_{f}, \quad \chi(0)=x_{0} .
\end{aligned}
$$

where $\alpha=b-f\left(s^{*}\right)$ and $b=\varpi+\rho$.

We substitute $\chi$ from (10) into (8), set $s=s^{*}$, and rearrange to isolate $f\left(s^{*}\right)$, to obtain

$$
\alpha=b-f\left(s^{*}\right)=b\left(\frac{x_{f}}{s_{f}+x_{f}-s^{*}}\right),
$$

and since $s^{*}<s_{f}+x_{f}$, we deduce $\alpha>0$.

To see that the linear system has the same critical point as the nonlinear system, we need only substitute the critical point $\left(s^{*}, x^{*}\right)$ from the nonlinear system into (16) and (17).

Since the Jacobian $J(s, x)$ of the right hand sides of (16)-(17) has eigenvalues $-\alpha,-\omega$, the one critical point $\left(s^{*}, x^{*}\right)$ of this system is always a stable node. However, as noted above, as a critical point of the nonlinear system (5)-(6), $\left(s^{*}, x^{*}\right)$ may be a stable node or spiral point. To preserve the nature of $\left(s^{*}, x^{*}\right)$ and to enhance the ability of the linearized system to accurately reflect the nonlinear system, we require, as above, that $\left[\operatorname{tr} J\left(s^{*}, x^{*}\right)\right]^{2}-4 \operatorname{det} J\left(s^{*}, x^{*}\right)>0$.

\section{Exact solutions of the linearized system}

Standard methods give the solution of the initial value problem (16)-(17) as

$$
\begin{aligned}
& x(t)=E_{0} e^{-\alpha t}+\frac{\varpi x_{f}}{\alpha}, \\
& s(t)=E_{1} e^{-\varpi t}+s_{f}+\frac{f\left(s^{*}\right)}{\rho-f\left(s^{*}\right)} E_{0} e^{-\alpha t}-\frac{x_{f} f\left(s^{*}\right)}{\alpha},
\end{aligned}
$$


where

$$
\begin{aligned}
& E_{0}=x_{0}-\frac{\varpi x_{f}}{\alpha} \\
& E_{1}=s_{0}-s_{f}-\frac{f\left(s^{*}\right)}{\rho-f\left(s^{*}\right)} E_{0}+\frac{x_{f} f\left(s^{*}\right)}{\alpha},
\end{aligned}
$$

and $\alpha$ is given by (18). Note that (19)-(20) requires that $\rho-f\left(s^{*}\right) \neq 0$. Since $\alpha>0$, this solution will always approach a finite limiting state, namely

$$
x^{*}=\frac{\varpi x_{f}}{\alpha}, \quad s^{*}=s_{f}-\frac{x_{f} f\left(s^{*}\right)}{\alpha} .
$$

A calculation shows that the critical points above agree with those of the original nonlinear system.

When $\rho-f\left(s^{*}\right)=0$, so that $\alpha=\varpi$, the solution of (16)-(17) becomes

$$
\begin{aligned}
& x(t)=K_{0} e^{-\varpi t}+x_{f}, \\
& s(t)=K_{1} e^{-\varpi t}+s_{f}-\frac{f\left(s^{*}\right) x_{f}}{\varpi}-K_{0} f\left(s^{*}\right) t e^{-\varpi t},
\end{aligned}
$$

where

$$
K_{0}=x_{0}-x_{f}, \quad K_{1}=s_{0}-s_{f}+\frac{f\left(s^{*}\right) x_{f}}{\varpi} .
$$

As before, since $\varpi>0$, this solutions will approach the limiting states (23).

\section{Discussion}

We now compare the numerical solutions of the original nonlinear system to the exact analytic solutions of the corresponding linearized system. The nonlinear system is linearized in a way that ensures that the critical points of the linear and nonlinear systems are the same. We assume that the initial conditions are physically real and therefore $s_{0} \geqslant 0, x_{0} \geqslant 0$. 
Although the solutions of (5) and (6) will always remain in the positive quadrant, $s(t) \geqslant 0, x(t) \geqslant 0$, if $s_{0} \geqslant 0, x_{0} \geqslant 0$, then this is not the case for the linear system. To see that $x(t) \geqslant 0$ for $t \geqslant 0$, note that $x(t)$ cannot become negative if $x_{0} \geqslant 0$. To see this, assume the system evolves to the point $x(t)=0$, then $x$ can only become negative if $d x / d t<0$ at this point; however, setting $x=0$ in (17) we see that $d x / d t=\varpi x_{f}>0$, so $x(t)$ can never be negative. However, it is possible, under certain circumstances, for $s(t)$ to become negative, which is clearly not physically realistic. Examining the right hand sides of (16) and (17) shows us that trajectories may only leave the first quadrant along that segment of the $x$ axis for which $\varpi s_{f}-f\left(s^{*}\right) x<0$. In fact, for a trajectory originating in the first quadrant to cross the $x$ axis, there must be a $t^{*}>0$ such that the four conditions

$$
s\left(t^{*}\right)=0, \quad d s / d t\left(t^{*}\right)<0, \quad \varpi s_{f}-f\left(s^{*}\right) x\left(t^{*}\right)<0, \quad d x / d t\left(t^{*}\right)<0
$$

hold. These provide a necessary condition on the parameters and initial conditions of the linearized problem for the existence of a non-physical solution.

However, even if solutions to the linear system are physically realistic for $t \geqslant 0$, it does not follow that they will always approximate well those of the nonlinear system. Numerical experiment shows that for values of $b>1$ the solutions from the linear system approximate those of the nonlinear system, and that the approximation improves the larger $b$ becomes. To see why this is so we consider the differential equations for the differences $x(t)-x_{1}(t)$ and $s(t)-s_{1}(t)$, where the subscript 1 denotes the solutions of the linear system and no subscript those of the nonlinear system.

The differential equations that govern $s(t)-s_{1}(t)$ and $x(t)-x_{1}(t)$ are

$$
\begin{aligned}
& \frac{d\left(s-s_{1}\right)}{d t}=-\varpi\left(s-s_{1}\right)-f\left(s^{*}\right)\left(x-x_{1}\right)-\left(f(s)-f\left(s^{*}\right)\right) x, \\
& \frac{d\left(x-x_{1}\right)}{d t}=-\alpha\left(x-x_{1}\right)+\left(f(s)-f\left(s^{*}\right)\right) x .
\end{aligned}
$$

Since $x, x_{1} \rightarrow x^{*}$ and $s, s_{1} \rightarrow s^{*}$ as $t \rightarrow \infty, s-s_{1}$ and $x-x_{1}$ go to zero, and the right hand sides of (27) and (28) go to zero as $t \rightarrow \infty$, convergence is 
not an issue. However, if the solutions of the linear system are to provide a reasonable approximation for those of the nonlinear system, then we require the $s-s_{1}$ and $x-x_{1}$ to be close to zero for most of $t>0$; that is, we require the convergence of $s-s_{1}$ and $x-x_{1}$ to be as rapid as possible. The question then arises, how might the parameter values and initial conditions affect this rate of convergence?

In (27) the only parameter is $\varpi>0$, and increasing its value will increase the rate of convergence of $s-s_{1}$ to zero. Looking at (28), similarly we see that increasing $\alpha$ will increase the rate of convergence of $x-x_{1}$. In addition, it is straightforward to show that $\alpha=b-f\left(s^{*}\right)$ is a monotonically increasing function of $b$, which increases more rapidly after $b=1$, so increasing $b$ will always increase the rate of convergence of $x-x_{1}$. So, the observation that convergence is rapid for $b>1$ and increases $a s$ increases is borne out by this analysis.

The initial conditions also have an effect on convergence. When the linear and nonlinear systems both start with the same initial conditions, $x(0)=x_{1}(0)$ and $s(0)=s_{1}(0)$, the initial rates of change in (27) and (28) are given by $\mp\left[f(s(0))-f\left(s^{*}\right)\right] x(0)$.

Figures 1 to 3 show comparisons between the solutions of the linearized system (16)-(17) and numerical solutions of the nonlinear system (5)-(6) for varying $b$ values with all other data constant. Figure 1 for $b=1.5$ shows very good agreement over all of $t \geqslant 0$. In Figure $2, b=1.01$ and the effect of this is shown in some discrepancies between plots, particularly in the transient region near $t=0$. Figure 3 , with $b=0.85$, shows marked differences between numerical and analytic solutions over most of $t \geqslant 0$.

These results support our discussion above regarding the role of $b$ in agreement between solutions of the nonlinear and linear systems.

Clearly, there are many aspects of the approach to the problem (16)-(17) that need investigation; and these are currently being explored by the first author. 
Figure 1: Linear and nonlinear system solutions with $\rho=0.8, \varpi=0.7$, $b=1.5, \alpha=0.642, s_{f}=7.0, x_{f}=0 ., x_{0}=3.0$ and $s_{0}=8.0$.

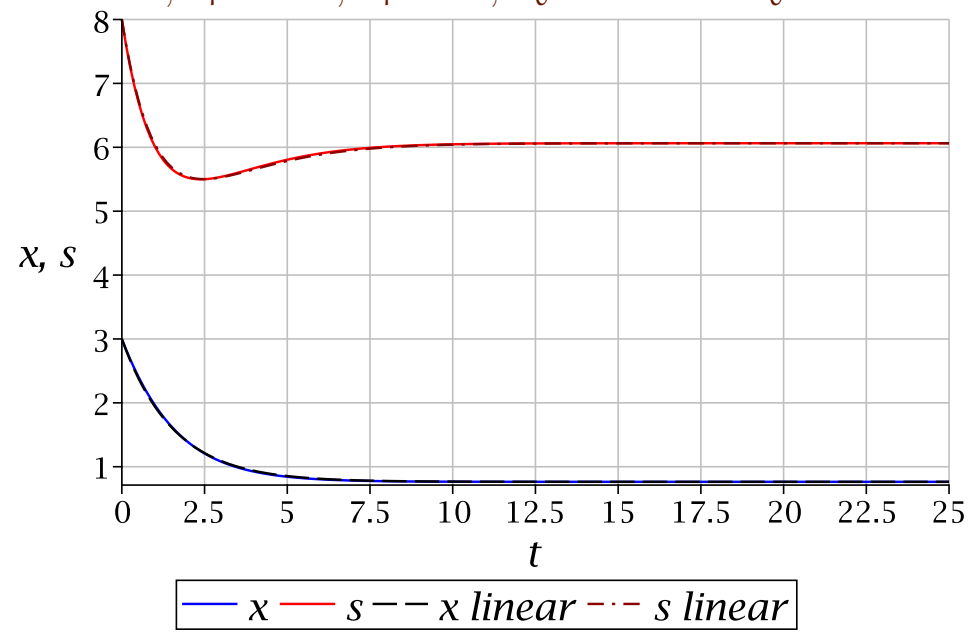

Figure 2: Linear and nonlinear system solutions with $\rho=0.31, \varpi=0.7$, $b=1.01, \alpha=0.202, s_{f}=7.0, x_{f}=0.7, x_{0}=3.0$ and $s_{0}=8.0$.

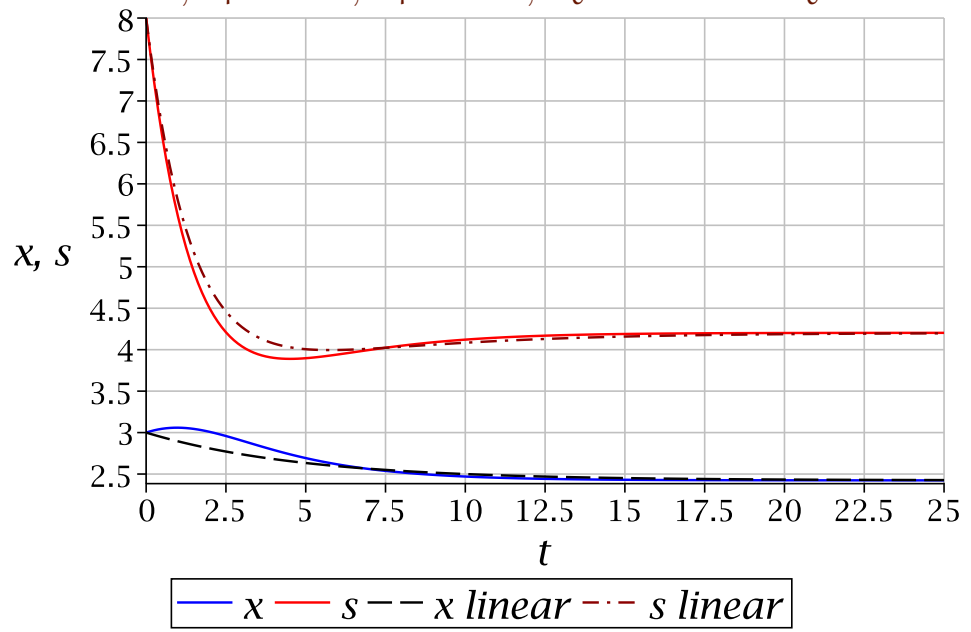


Figure 3: Linear and nonlinear system solutions with $\rho=0.5, \varpi=0.35$, $b=0.85, \alpha=0.119, s_{f}=7.0, x_{f}=0.7, x_{0}=3.0$ and $s_{0}=8.0$.

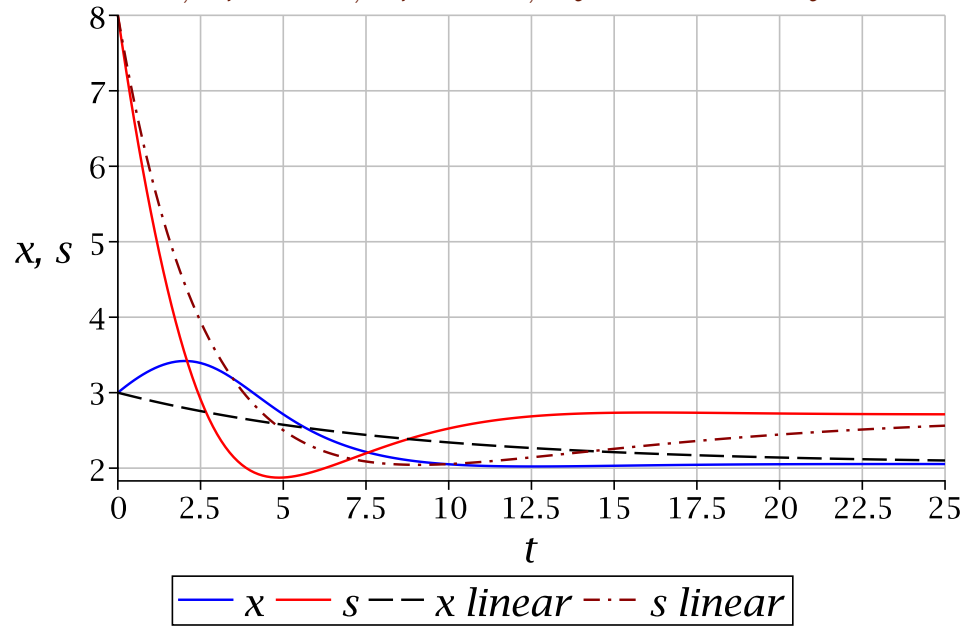

\section{References}

[1] J. E. Bailey and D. F. Ollis. Biochemical Engineering Fundamentals. McGraw-Hill Book Company, New York, 1966 (cit. on p. C231).

[2] D. J. Batstone, J. Keller, I. Angelidaki, S. V. Kalyuzhny, S. G. Pavlostathis, A. Rozzi, W. T. M. Sanders, H. Siegrist, and V. A. Vavilin. "The IWA anaerobic digestion model No 1 (ADM1)". In: Water Sci. Tech. 45.10 (2002), pp. 65-73. DOI: 10.2166/wst.2002.0292 (cit. on p. C230).

[3] D. T. Hill. "Simplified Monod kinetics of methane fermentation of animal wastes". In: Agri. Wastes 5.1 (1983), pp. 1-16. DOI: 10.1016/0141-4607 (83)90009-4 (cit. on p. C230).

[4] J. Monod. "The growth of bacterial cultures". In: Ann. Rev. Microbio. 3.1 (1949), pp. 371-394. DOI: 10.1146/annurev.mi.03.100149.002103 (cit. on p. C230). 
[5] F. E. Mosey. "Mathematical modelling of the anaerobic digestion process: Regulatory mechanisms for the formation of short-chain volatile acids from glucose". In: Water Sci. Tech. 15.8-9 (1983), pp. 209-232. DOI: 10.2166/wst.1983.0168 (cit. on p. C230).

[6] J. Rodriguez, E. Roca, J. M. Lema, and O. Bernard. "Determination of the adequate minimum model complexity required in anaerobic bioprocesses using experimental data". In: Chem. Tech. Biotech. 83.12 (2008), pp. 1694-1702. DOI: 10.1002/jctb.1990 (cit. on p. C230).

[7] H. L. Smith and P. Waltman. The Theory of the Chemostat: Dynamics of Microbial Competition. Cambridge University Press, 1995. DOI: 10.1017/CB09780511530043 (cit. on p. C231).

\section{Author addresses}

1. A. K. Alsharidi, Mathematical Sciences, School of Science, RMIT University, Melbourne, Australia and Department of Mathematics, Al Imam Muhammad Bin Saud Islamic University (IMSIU), Riyadh, SAUdi ARABia.

2. J. J. Shepherd, Mathematical Sciences, School of Science, RMIT University, Melbourne, Australia. mailto: john. shepherd@rmit.edu.au

3. A. J. Stacey, Mathematical Sciences, School of Science, RMIT University, Melbourne, Australia.

4. A. A. Khan, Mathematical Sciences, School of Science, RMIT University, Melbourne, Australia. 